Instituto Internacional de Investigación y Desarrollo Tecnológico Educativo INDTEC, C.A.

DOI: https://doi.org/10.29394/scientific.issn.2542-2987.2016.1.1.7.115-133

OAI-PMH: http://www.indteca.com/ojs/index.php/Revista Scientific/oai

\title{
Manejo Integral de Desechos Sólidos Mediante el Compostero
}

\author{
Autora: Griselda del Carmen Navarro Gutiérrez \\ Universidad Pedagógica Experimental Libertador, UPEL \\ griseldanavarrog@gmail.com; griseldanavarro1@hotmail.com \\ Barinas, Venezuela
}

\section{Resumen}

Los residuos y desechos sólidos se han convertido en el principal problema ambiental de la población. De allí se originan problemas como la contaminación del agua, aire y suelos, porque los desechos actúan como un factor desencadenante y vulnerable como lo es la basura. Una alternativa para dar solución a esta problemática es el reciclaje de estos desechos sólidos. Esta investigación tiene como propósito general Promover un Manejo Integral de Desechos Sólidos mediante el compostero en la "U.E.N Herminio León Colmenarez", en Barinas Estado Barinas. Este estudio se enmarcó en una Investigación Acción Participativa (IAP), y es bajo una naturaleza de investigación cualitativa, cimentada en la modalidad tipo descriptiva, basado en un diseño de campó. Para la investigadora las principales técnicas consideradas es la observación directa y participante, la realización de un guión de entrevista programada, con preguntas en orden de redacción para los entrevistados, se analizan las respuestas de la entrevista a profundidad y se establecerán las categorías y sub categorías, se mostrarán los hallazgos, para realizar las respectivas conclusiones y recomendaciones. Con la finalidad de darles un manejo integral a los desechos sólidos, a través del compostero, como una manera de activar acciones ambientalistas en pro de la conservación del ambiente.

\footnotetext{
Palabras clave: desechos sólidos; reciclaje; compostero; educación ambiental.
} 


\title{
Management Integral of Solid Wastes Through the Composting
}

\begin{abstract}
Waste and solid wastes have become the main environmental problem of the population. From there are generated problems such as pollution of water, air and soil arise because the wastes act as a trigger factor and vulnerable as it is trash. An alternative to solve this problem is the recycling of these solid wastes. This research has the general purpose to promote a Comprehensive Solid Waste Management by composting in the "U.E.N Herminio León Colmenarez" in Barinas State. This study was part of a Participatory Action (IPA) Research, and it is under nature of qualitative research, grounded in descriptive form, based on a field investigation. To researcher the main techniques considered is the direct observation and participant performing a script scheduled interview, with questions by writing to the respondents, the answers of the interviews are deeply analyzed and categories and sub categories will be stablished, the findings will be showed, for doing the respective conclusions and recommendations. In order to give them a comprehensive management of solid wastes through composting as a way to activate environmental actions for environmental conservation.
\end{abstract}

Keywords: solid wastes; recycling; composting; environmental education. 


\section{Modo de Introducción}

La contaminación del ambiente ocasionada por las actividades antropológicas es un problema complejo y de proporciones mundiales debido a sus políticas, económicas, científicas, tecnológicas y culturales que interactúan de una manera tan estrecha que muchas veces dificultan la ejecución de acciones correctivas a nivel local, regional y nacional debido a que prácticamente no hay un lugar en el planeta quien escape a sus consecuencias.

Así mismo la basura es un gran problema de todos los días y un drama terrible para las grandes ciudades ya que no saben qué hacer con tantos desperdicios que son fuente de contaminación ambiental, malos olores, y además producto de la proliferación de insectos, roedores y microorganismos patógenos, trayendo como consecuencia enfermedades catastróficas para el hombre.

Actualmente se experimenta en el mundo un rápido crecimiento poblacional, lo cual conlleva ciudades más grandes, con mayor consumo y más generadoras de residuos, es por ello que el reciclado de residuos permite disminuir los riesgos de contaminación y en el caso particular de los residuos domiciliarios permite reducir la cantidad que se deriva a rellenos sanitarios 0 depósitos a cielo abierto, promoviendo prácticas tendientes a conservar los recursos naturales.

\subsection{Contextualización de la Situación}

El hombre como parte integral de un sistema de diversidad actúa sobre la naturaleza y crea su propia cultura, con el paso del tiempo y como resultado de la transformación del ambiente y las interrelaciones sociales, que van dejando huella de sus acciones, creándose así la basura o residuos sólidos como un problema alarmante en cualquier comunidad local, regional, nacional y mundial. 
Es por ello, que el manejo de los desechos sólidos generados por el mismo constituye uno de los aspectos más trascendentales y fundamental que afecta la salud pública, que de no ser realizado adecuadamente originan problemas ambientales y de salud de gran magnitud a un corto, mediano o largo plazo, alterando la salud y calidad de vida de las personas y a su vez deterioran el ambiente.

En este sentido, la Organización Mundial de la Salud (1999), en el marco de la "Asamblea Mundial de la Salud" celebrada en el año 1977 y en la "Declaración de 1978 de Alma-Ata" (URSS) sobre la Atención Primaria de la Salud, le confirió especial importancia a los efectos de las interacciones básicas de los factores ambientales y la salud, por lo cual recomienda a sus países miembros la adopción de políticas y estrategias para la gestión de desechos, destinadas a tratar dicho fenómeno de manera integral y adecuada a la realidad de cada país.

Es importante resaltar que en la actualidad el compostaje se ha convertido en una alternativa para el manejo de los desechos sólidos ya que el mismo consiste básicamente en la transformación mediante la fermentación controlada, de la materia orgánica fermentable presente en los residuos urbanos con la finalidad de obtener un producto inocuo y con buenas propiedades como fertilizante o enmienda orgánica de suelos que recibe el nombre de compost o abono orgánico. Según Haug (1993) define el compostaje como:

La descomposición y estabilización biológica de substratos orgánicos, bajo condiciones que permitan el desarrollo de temperaturas termófilas como resultado del calor producido biológicamente, para producir un producto final estable, libre de patógenos y semillas que puede ser aplicado de forma beneficiosa al suelo (pág. 54)

Lo anterior induce a pensar que durante el proceso de compostaje los microorganismos se transforman en residuos orgánicos, es por ello que se 
pueden utilizar para procesar y dar un mayor uso a los desechos sólidos, en especial los de origen orgánico que se generan a nivel, local, regional y nacional.

Es por ello que el manejo de los residuos sólidos es una problemática ambiental que se ha profundizado en las últimas décadas. Debido a que toda actividad humana potencialmente es susceptible de producir residuos, estos pueden ser producto de las actividades minera, agricultura y doméstica, además de producción de energía.

Los residuos sólidos se definen como todos los residuos domésticos y los desechos no peligrosos, como los desechos comerciales e institucionales, industrias y establecimientos de salud, las basuras de la calle y los escombros de la construcción, que normalmente son sólidos a temperatura ambiente. Al respecto, Castillo, (2007), señala:

Durante el último siglo la acumulación, de residuos sólidos se ha convertido en un problema ambiental que debe ser atendido, con urgencia. Cada día, en las grandes ciudades del mundo, se generan toneladas de basura, generando costos sociales y económicos crecientes asociados a manejo y disposición final (pág. 3.).

Es decir, que mientras más industrializada, más urbanizada y más desarrollada es una sociedad, la cantidad de basura por persona, también ha ido en aumento.

Día a día se observan grandes cantidades de desechos sólidos, amontonados o regados en lugares menos indicados, como en los planteles e instituciones a nivel local, regional o nacional desmejorando el ambiente y con ello la naturaleza.

En vista de la situación antes descrita y al considerar que la misma desde un punto de vista sanitario representan un alto riesgo para la alteración de la salud y el bienestar de los habitantes expuestos ante esta realidad. 
En donde esta problemática que se describe ocurre en todo el todo territorio nacional, a lo cual no escapa el Estado barinas, particularmente en la comunidad Dominga Ortiz de Páez, en la U.E.N. "Herminio León Colmenarez" donde se observa que los desechos sólidos originados por el Programa de Alimentación Escolar Bolivariana (PAEB), se han convertido en una fuente de contaminación dentro de la misma institución. En este mismos orden de ideas y ante la situación ya descrita, se cree conveniente que a través del uso del compost, darles un mejor uso y adecuado procesamiento de los desechos sólidos descritos al propiciar la elaboración de fertilizante y a su vez ser utilizado para realizar la siembras de cilantro, cebollín y ají dulce en el mismo plantel y así incentivar a la comunidad educativa en general a realizarlos en sus hogares y darles otra utilidad a estos residuos sólidos y transfórmalos en materia orgánica provechosa.

Es por ello, que surge la motivación de la investigadora para realizar un estudio acerca del manejo integral de los desechos sólidos, por parte de los habitantes de la comunidad de la Dominga Ortiz de Páez, comenzando en la Institución educativa ya mencionada, en los hogares y en las zonas aledañas a la misma, a los fines de proponer una alternativa efectiva para solventar la problemática descrita, razón por la cual con la presente investigación se propone dar respuesta a los siguientes interrogantes:

¿Qué conocimientos poseen la población estudiantil y comunidad sobre los desechos sólidos? ¿Está la población estudiantil y comunidad sensibilizada para clasificar los desechos sólidos? ¿Cómo podemos utilizar y reciclar los residuos sólidos? ¿Qué utilidad le podemos dar a los desechos sólidos?

¿Cómo integrar a la comunidad en general en pro de la conservación y protección del ambiente? ¿Cómo promover el uso del compostero?

Para dar respuestas a dichas interrogantes se formula el Propósito de General de la Investigación, Promover un Manejo Integral de Desechos Sólidos mediante el compostero en la U.E.N. "Herminio León Colmenarez", en 
Barinas Estado Barinas. Esta investigación se estableció como alternativa a la solución de la problemática ambiental sobre los desechos sólidos a través de la elaboración de compostero en este plantel y en la comunidad en general, cuya ejecución contribuye a mejorar la calidad de vida de los habitantes.

Es importante resaltar que este trabajo responde a la línea de investigación: Educación, Desarrollo y Gestión Comunitaria de la Universidad Pedagógica Experimental Libertador (UPEL).

\section{Teoría y Conceptos}

\subsection{Antecedentes Investigativos}

Esta sesión se refiere a los estudios anteriores, representados por tesis de grado, postgrado, doctorales, informes científicos y otros trabajos de investigación relacionados con el problema, es decir, la revisión de investigaciones previas relacionadas de manera directa o indirecta con el problema en estudio. Por su parte Claret (2006), expresa que "se refiere a la revisión de trabajos previos sobre el tema en estudio realizado fundamentalmente en instituciones de educación superior reconocidas o, en su defecto, en otras organizaciones" (pág. 19). En consecuencia, su revisión debe hacerse partiendo de las publicaciones más antigua a la más actual.

En este mismo orden de ideas Guerra y Pérez (2010), en su trabajo de investigación que lleva por nombre "Diagnóstico, Manejo y Disposición de los Desechos sólidos generados por la comunidad Boyaca III sector oeste, municipio Simón Bolívar. Estado Anzoátegui", Ingeniería Civil, de la Universidad de Oriente, Núcleo Anzoátegui, Escuela de Ingeniería y Ciencias Aplicadas, Departamento de Ingeniería Civil, cuyo propósito parte de Evaluar el sistema de recolección, manejo y disposición de los desechos sólidos generados por la comunidad Boyacá III Sector Oeste, Municipio Simón Bolívar, Estado Anzoátegui. 
De igual forma Martínez y Herrera (2010), en su trabajo de grado sobre un "Diagnóstico del Sistema de Recolección, Manejo y Disposición de los Desechos Sólidos generados por la comunidad "El Esfuerzo", Municipio Simón Bolívar, Estado Anzoátegui, de la Universidad de Oriente Núcleo de Anzoátegui Escuela de Ingeniería y Ciencias Aplicadas. Departamento de Ingeniería Civil, con el propósito de Evaluar el sistema de recolección, manejo y disposición de los desechos sólidos generados por la comunidad El Esfuerzo, Municipio Simón Bolívar, Estado Anzoátegui.

Igualmente Villegas (2011), realizó un trabajo sobre una "Propuesta para la capacitación de la población estudiantil en la elaboración de compost, en la Unidad Educativa Francisco Javier Urbina" de Flor de Patria Municipio Pampán Estado Trujillo, de la Universidad de los Andes Núcleo Universitario "Rafael Rangel" departamento de Biología y Química Trujillo Estado Trujillo, con el propósito de proponer estrategias para capacitar a los estudiantes de Quinto grado de la Unidad Educativa Francisco Javier Urbina" de Flor de Patria, municipio Pampá, Estado Trujillo, sobre la elaboración del compost .

\subsection{Referentes Teóricos}

El marco conceptual que a continuación se presenta, sirve de referencia al planteamiento sobre una educación ambiental para el manejo integral de los desechos sólidos de origen orgánico mediante el compostero en la U.E.N. "Herminio León Colmenarez". En este orden de ideas se exponen las siguientes definiciones:

\section{Manejo Integral de los desechos sólidos}

Es aquel que incluye un conjunto de planes, normas y acciones para asegurar que todos sus componentes sean tratados de manera ambientalmente adecuada, técnica y económicamente factible y socialmente aceptable. Según (Pineda, 1998, pág. 77), "el manejo en el origen se refiere a 
actividades con el manejo de los desechos sólidos hasta que son depositados en recipientes utilizados para el almacenamiento antes de la recolección". Dependiendo del tipo de servicio de recolección y para devolver los recipientes vacíos al lugar donde se colocan entre las recolecciones.

\section{Desechos sólidos}

Los desechos sólidos son los que ocupan un mayor porcentaje en el total de desechos o residuos que el ser humano genera debido a que gran parte de lo que se consume o se utiliza en la vida cotidiana dejan desechos de este tipo. Además, los desechos sólidos son también los que ocupan mayor espacio al no asimilarse al resto de la naturaleza y al permanecer muchos de ellos por años e incluso siglos en el terreno.

Tal como lo establece (Corbitt, 1999, pág. 78), los desechos sólidos "comprenden de manera general, las basuras domésticas y todos los desperdicios sólidos provenientes de actividades comerciales, industriales y agrícolas, que afectan directamente al paisaje y con ello el ambiente".

\section{Tipos de desechos}

Así mismo la basura se clasifica de acuerdo con el tipo de material de desecho, que puede ser orgánico e inorgánico. Tomando en consideración lo planteado anteriormente. Flores, (1998), expresa, que los desechos sólidos pueden ser de varios tipos, entre los cuales cabe mencionar: "1. Los desechos orgánicos provienen de la materia viva e incluyen restos de alimentos, papel, cartón y estiércol. 2. Los desechos inorgánicos, provienen de la materia inerte como: el vidrio, los plásticos, los metales y otros materiales" (pág. 60).

Este mismo autor señala que la basura también se puede clasificar según el tiempo en que los materiales tardan en degradarse por la acción de los organismos descomponedores llamados bacterias y hongos. 


\section{Así los desechos se clasifican en:}

Los Desechos Biodegradables son los que se descomponen de forma natural en un tiempo relativamente corto.

Por ejemplo: los desechos orgánicos, como los alimentos que tardan poco tiempo en descomponerse.

\section{Los Desechos No Biodegradables}

Son los que no se descomponen fácilmente, sino que tardan mucho tiempo en hacerlo.

Por ejemplo: el vidrio tarda unos 4000 años, el plástico tarda de 100 a 1000 años, una lata de refresco tarda unos 10 años, un chicle 5 años.

De igual manera la fundación Vitalis (2000), divide los desechos sólidos en:

Orgánica: Comprende los residuos provenientes de restos de alimentos, desperdicios de origen animal, vegetal y productos como: papel, cartón, cuero o madera.

Inorgánica: Formada por material inerte $\circ$ material, que se descompone muy lentamente. Incluye los escombros de construcción, chatarra y restos de actividades, mineras entre otras.

Residuos Especiales: Engloba los platicos, vidrios, cauchos sintéticos otros residuos como los plaguicidas a base de cloro, el DDT y los desechos de industrias químicas, petroquímicas y de fundación.

\section{El compostado}

"Es sinónimo de descomposición biológica. Es un método mediante el cual los componentes orgánicos de los desechos son biológicamente descompuestos, bajo condiciones controladas, obteniéndose un producto que puede ser almacenado y aplicado al suelo sin efectos adversos al medio". Tal como lo expresa el Instituto de (Recursos Naturales Renovables de la 
Universidad Simón Bolívar, 1981, pág. 56). Es decir, que el abono obtenido es un buen fertilizante. Desde el punto de vista sanitario la compostación es un método aceptable.

\section{Metodología}

\subsection{Naturaleza de la Investigación}

Llegada a esta sesión del trabajo, en cuanto al diseño del marco metodológico, para (Hurtado, et al., 2007, pág. 90), "constituye la médula de la investigación"; en tal sentido, se indican los procesos que se llevaron a cabo para ejecutar la indagación.

La investigación está centrada dentro del enfoque cualitativo, el cual está basado en los siguientes fundamentos que, según (Martínez, et al., citado por Aponte, 2001), "trata de identificar la naturaleza profunda de la realidad, su estructura dinámica, aquella que da razón plena de su comportamiento y manifestaciones".

Parafraseando lo anterior el investigador realiza el estudio desde afuera, pero integrando a cada miembro de la comunidad para establecer los problemas y sus posibles soluciones.

La finalidad de la IAP es cambiar la realidad y afrontar los problemas de una población a partir de sus recursos y su participación.

\subsection{Tipo de investigación}

Con respecto al tipo de investigación, se utilizará la investigación acción participativa, la cual, es un método de estudio y acción de tipo cualitativo que busca obtener resultados fiables y útiles para mejorar situaciones colectivas, basando la investigación en la participación de los propios colectivos a investigar. 
Según Ander, E. (1990), define, que el método de la investigación acción participación (IAP) combina dos procesos, el de conocer y el de actuar, implicando en ambos a la población cuya realidad se aborda.

Esta investigación se realizará según el modelo metodológico ofrecido por Hurtado y Toro (ob.cit.), donde se propone un plan general de investigación contemplando las siguientes fases:

1. Un Diagnostico, el cual lleva a la identificación de los procesos problemas que quiere investigar.

2. Una Planificación, delimitación de lo que se quiere cambiar (procedimientos de la enseñanza ejemplificando), descripción de las estrategias y propuestas para la acción inicial.

3. Una Ejecución, que contempla la descripción de cómo se ejecutaron las estrategias previstas, descripción del proceso de registro de la ejecución de las estrategias, incluyendo técnicas e instrumentos, a través de los cuales se recoge la información.

4. La Evaluación, valorización de las acciones ejecutadas, construcción teórica (tema de estudio, acción, información), preparación de las conclusiones y reflexiones de la situación problema que se quiere estudiar, siendo el proceso de construcción permanente.

5. Así mismo, inserta como fase de la investigación acción la sistematización, el cual consiste en la reconstrucción del trabajo que permita "tener una visión más global y profunda de la práctica", con el fin de obtener conclusiones que servirán en el futuro.

\subsection{Diseño de la investigación}

El diseño de la investigación es de campo según el autor Santa, et al., 2010, pág. 88), define: "La Investigación de campo consiste en la recolección de datos directamente de la realidad donde ocurren los hechos, sin manipular o controlar las variables. Estudia los fenómenos sociales en su ambiente 
natural". Parafraseando lo anterior el diseño de la investigación no es más que la manera de cómo e investigador recolecta la información de los actores sociales en el ambiente sin investigar las variables.

\subsection{Informantes Claves}

(Martínez, 1998, pág. 7), que se trata de "reportes de investigaciones basados en personas que aportan la información, enfatizando en quienes son y no sobre cuántos son". Es por ello que, en todo el proceso se busca establecer una relación de confianza con los informantes a objeto de recabar la información necesaria y de interés para la investigación. Parafraseando lo anterior los informantes claves son personas que hacen vida activa en la comunidad afectada y poseen información relevante para el estudio de la problemática a tratar.

\subsection{Técnica e instrumentos de recolección de datos}

Según (Hurtado, 2008, pág. 153), la técnica de recolección de datos comprende procedimientos y actividades que le permiten al investigador obtener la información necesaria para dar respuesta de su investigación.

Las técnicas de observación, redacta (Zapata, 2006, pág. 110), que "son procedimientos que utiliza el investigador para presenciar directamente el fenómeno que estudia sin actuar sobre el esto es, sin modificarlo o realizar cualquier tipo de operación que permita manipular". Así mismo para (Rojas, 2001, pág. 61), una guía de observación es un conjunto de preguntas elaboradas con base en ciertos objetivos e hipótesis y formuladas correctamente a fin de orientar nuestra observación. Estos instrumentos permiten registrar los datos en orden cronológico, practico y concreto para derivar de ellos el análisis de una situación o problema determinado.

La entrevista según (Pardinas, 1991, pág. 115), refleja que la técnica consiste simplemente en plantear las preguntas tan rápidamente como el 
entrevistado sea capaz de responder y de comprender. Se elaborará como instrumento, una guía de entrevista de semi estructuradas, relacionadas con las categorías y sub categorías descritas en el estudio, las cuales permitirán ofrecer respuestas abiertas proporcionadas según el pensamiento y conocimientos de los informantes claves de la comunidad. Parafraseando los párrafos anteriores las técnicas de recolección de datos permitirán al investigador recabar toda la información necesaria para realizar la interpretación o análisis de la información.

\section{Consideraciones finales y Recomendaciones}

\subsection{Análisis de los Resultados}

Dentro del enfoque cualitativo, existe una serie de etapas principales en el análisis e interpretación de los resultados, donde es preciso resaltar, que estas descripciones intimas presentan detalladamente el contexto y los significados de los acontecimientos y escenas importantes para los involucrados.

Según el análisis realizado al dialogo semi estructurado en las entrevistas con los informantes claves que fue a 7 personas cabezas de familias, representantes y a la observación directa desarrollada por la investigadora en la U.E.N. "Herminio León Colmenarez", parroquia Ramón Ignacio Méndez, en donde estos arrojaron datos significativos y se obtuvieron los siguientes resultados, en el estudio tal y como se explicó en la parte metodológica debido a que fue llevado a cabo mediante la indagación y la aplicación del instrumento por medio de la entrevista para recabar la información o datos suministrados por cada uno de los y las informantes claves.

Cabe destacar que todos los procedimientos metodológicos permitieron realizar de manera progresiva una hermeneusis de la situación objeto de estudio para de esta forma se organizaran y jerarquizaran las dimensiones 
para el posterior análisis cualitativo de la información obtenida la cual se reordeno siguiendo las fases, que ofrece el modelo metodológico citado por Hurtado y Toro (ob.cit.), las cuales llevan una secuencia en la investigación como son las siguientes: 1. Diagnóstico, nos permite conocer los problemas de nuestra comunidad. 2. Planificación, nos permite preparar las acciones para solucionar los problemas detectados. 3. Ejecución, nos permite realizar las acciones planificadas. 4. Evaluación, nos permite valorar las acciones que hemos realizado. 5. Sistematización, nos permite reconstruir las experiencias vividas de todo el proceso de trabajo.

\subsection{CONCLUSIÓN}

Este trabajo de investigación tuvo un gran impacto en la U.E.N. "Herminio León Colmenarez", en vista que fueron receptivos sobre la problemática que se está viviendo actualmente por los desechos sólidos generados por el Programa de Alimentación Escolar Bolivariana (PAEB), con la finalidad de darles otro uso a través de un manejo integral y a la clasificación de los residuos sólidos.

Por tal motivo se buscó la solución mediante el desarrollo de las actividades con los integrantes de la comunidad educativa para la realización de las mismas descritas en el plan de acción, debido a que se les dio instrucción a la población estudiantil y a la comunidad educativa, sobre el manejo integral de desechos sólidos, mediante la selección, clasificación y la elaboración del compostero, por medio de charlas y talleres de capacitación.

Así mismo se les dio a conocer las diferentes estrategias para la recolección y clasificación de los desechos sólidos en la escuela y en el hogar, con el fin de lograr una mayor interacción familia y comunidad, en pro de la conservación del ambiente.

De igual manera se propuso el compostero como alternativa agroecológica en el desarrollo sustentable de la institución educativa y de la 
comunidad en general, para proporciónale a los suelos nutrientes que nos garantice las siembras de rubros como: cilantro, cebollín, ají dulce, y pimentón.

Finalmente se motivó a la comunidad, consejo comunal y a los y las habitantes, a integrarse y a participar en las distintas actividades, para solventar la problemática en cuanto al manejo integral de los desechos sólidos mediante el compostero, con la finalidad de obtener un producto inocuo con buenas propiedades y que a su vez el mismo nos sirve como fertilizante.

\subsection{Recomendaciones}

Una vez finalizada la investigación se recomienda lo siguiente:

1). Mantener los recipientes que contienen los residuos tapados y en un lugar alejado de los animales. 2). Identificar los diferentes componentes que contienen los residuos y separar los orgánicos de los inorgánicos, ya que los mismos se pueden volver a usar o reciclar. 3). Si no existe un sistema de recolección, organicemos en conjunto con los representantes, vecinos y el consejo comunal, un sistema apropiado para su disposición sin causar deterioro en el ambiente. 4). Por ningún motivo no reutilicemos recipientes que hayan contenido sustancias peligrosas.

\section{Referencias}

Ander, E. (1990). Repensando la investigación Acción Participativa. Servicio de publicaciones del Gobierno Vasco. Vitoria.

Aponte, J. (2001). Juegos Ecológicos como Estrategias Didácticas para fomentar el espíritu cooperativo en la realización de trabajo grupal, en los alumnos de 3er grado sección C de la U.E "Ramón Francisco Feo". Trabajo de Pregrado. Calabozo, Guárico: Universidad Rómulo Gallegos.

Castillo, H. (2007). La Sociedad y el Trabajo de la Basura. Recursos, Conservación y Reciclaje. 39,193-210. 
Claret, A. (2006). Cómo hacer y defender una tesis. Quinta Edición. Caracas: Editorial Texto, C.A.

Corbitt, R. (1999). Manual de referencia de ingeniería ambiental. Bogotá, Colombia.

Flores, E. (1998). Ciencias Hoy. Caracas, Venezuela.

Guerra y Pérez (2010). Diagnóstico, Manejo y Disposición de los Desechos sólidos generados por la comunidad Boyaca III sector oeste, municipio Simón Bolívar. Estado Anzoátegui. Trabajo de Grado. Universidad de Oriente. NAEICADIC. Venezuela.

Haug, R. (1993). El Manual Práctico de Compostaje Engineering. Lewis Publishers. Nueva York.

Hurtado, I. y Toro, J. (2007). Paradigmas y Métodos de la Investigación en tiempos de cambios. Editorial CEC, S.A.

Hurtado de Barrera, J. (2008). El proyecto de investigación. (7ma. Edición). Caracas: Quirón.

Instituto de Recursos Naturales Renovables de La Universidad Simón Bolívar (1981). Ambiente Basura. Recirculación de Desechos Sólidos en Venezuela. 23(1), 53-57. Fundación de Educación Ambiental. Caracas, Venezuela.

Manual de Trabajo de Grado de Especialización y Maestría y Tesis Doctorales. (1998). FUNDAUPEL. Caracas, Venezuela.

Martínez. M, (1998). La Investigación Etnográfica en Educación. Manual Teórico Práctico. Tercera edición. México: Editorial Trillas.

Martínez y Herrera (2010). Diagnostico del Sistema de Recolección, Manejo y Disposición de los Desechos Sólidos, generados por la comunidad "El Esfuerzo. Municipio Simón Bolívar, Estado Anzoátegui. Trabajo de Grado. Universidad de Oriente NAEICADIC. Venezuela. 
Organización Mundial de la Salud (1999). Salud medio Ambiente y Desarrollo. Enfoques para la prevención de Estrategias a Nivel de países, para el bienestar humano según la Agenda 21. Washington, D.C.

Palella, S. y Martins, F. (2010). Metodología de la Investigación. (4ta ed.). Caracas: FEDUPEL.

Pardinas, E. (1991). Metodología y técnicas de investigación en ciencias sociales. Siglo Veintiuno. México.

Pineda, S. (1998). Manejo y disposición de los residuos sólidos urbanos. Bogotá, Colombia.

Villegas, Y. (2011). Propuesta para la capacitación de la población estudiantil en la elaboración de compost. En la Unidad Educativa "Francisco Javier Urbina", de Flor de PATRIA, Municipio Pampán. Estado Trujillo. Trabajo de Grado. Universidad de los Andes NURR. Venezuela.

Zapata, D. (2006). Restauración de la Plaza "Delia Ávila de Zapata", de la Urbanización El Valle, Municipio Bermúdez Estado Sucre. Trabajo de Grado. Universidad de Oriente. 


\section{Griselda del Carmen Navarro Gutiérrez}

e-mail: griseldanavarrog@gmail.com; griseldanavarro1@hotmail.com

Nacida en Barinas. Estado Barinas. Profesora en

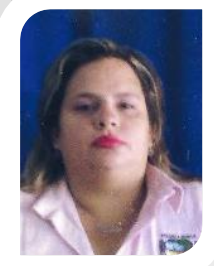

Educación Mención Integral, graduada en la Universidad Pedagógica Experimental Libertador (UPEL), Barinas. (2008). Soltera. Docente III. Estudiante de la Maestría en

Educación Ambiente y Desarrollo en la Universidad Pedagógica Experimental Libertador (UPEL), Barinas. Trabajo en el Ministerio del Poder Popular para la Educación (MPPPE). Pertenezco a la nómina y trabajo en la U.E.N. "Herminio León Colmenarez". Actualmente me desempeño como: Docente en Función: Coordinadora en el turno de la mañana del Programa de Alimentación Escolar Bolivariana (PAEB), Barinas Estado Barinas. 